Radial and Nonradial Pulsations as Probes of Stellar Physics

ASP Conference Series, Vol. 259, 2002

C. Aerts, T.R. Bedding, \& J. Christensen-Dalsgaard, eds.

\title{
Frequency Analysis of $\beta$ Cephei Stars in NGC 4755
}

\author{
A. Stankov \\ Institut für Astronomie, Universität Wien, Türkenschanzstr.17, 1180 \\ Wien, Austria
}

\begin{abstract}
We present the results of the multi-color, two-site observing campaign of $\beta$ Cephei stars in the young open cluster NGC 4755, from 1999 and 2000. More than 200 hours of time-series photometric $U, B$, $V$ filter data of the variable stars were acquired. We show a table of the detected frequencies and discuss the results.
\end{abstract}

\section{Introduction and observations}

$\beta$ Cephei stars are early B-type stars with periods between 2 and 7 hours. Their variability is caused by the classical $\kappa$-mechanism operating in the heavy-element ionization zones (Moskalik \& Dziembowski, 1992). Many of the $\beta$ Cephei stars are multi-mode pulsators, and radial as well as nonradial pulsation may occur.

In this project we apply multisite multi-color time series photometry for $\beta$ Cephei stars which proved to work very well for $\delta$ Scuti stars (e.g., Breger et al., 1998 and Stankov et al., 1998), in order to perform asteroseismology.

The observations were obtained with CCD detectors in three filters $(U, B$, $V$ ). The participating observatories were SSO (4 nights 1999 and 7 nights 2000) and SAAO (21 nights 2000). We used 40 hours from 1999 and 220 from 2000.

The frames were corrected for bias and flat-fielded using the standard IRAF reduction techniques. The photometry was performed using the program MOMF (Kjeldsen \& Frandsen, 1992). The pulsation frequencies were obtained by Fourier-analysis of the light curves using Period98 (Sperl, 1996). The results of the frequency analysis can be seen in Table 1 .

\section{Results and conclusions}

NGC 4755-F: Our observations show the presence of three frequencies.

NGC 4755-G: We detected four pulsation frequencies. Star G was discovered by Koen (1993) to be a $\beta$ Cephei variable with two frequencies. It is remarkable that in 1996 (Balona et al., 1997) this star did not appear to be pulsating but now the pulsation is detectable again.

NGC 4755-I: We were able to detect two pulsation frequencies. It seems as if the pulsation in this star had stopped between 1994 and 1996. Previous observations (Balona, 1994) showed it to have one of the largest amplitudes (7.3 mmag at $f=5.585 \mathrm{~d}^{-1}$ ) of the $\beta$ Cephei stars in NGC 4755 . We re-detected the pulsations in the 1999 data as well as in the 2000 data.

NGC 4755-201: Our observations resulted in the detection of one frequency 
Table 1. The frequency spectrum of the $\beta$ Cephei stars in NGC 4755. Identification numbers from Arp \& van Sant (1958).

\begin{tabular}{|c|c|c|c|c|}
\hline Star & $\begin{array}{c}\text { Frequency } \\
\qquad d^{-1}\end{array}$ & $U \mathrm{mmag}$ & $\begin{array}{c}\text { Amplitude } \\
B \text { mmag }\end{array}$ & $V$ mmag \\
\hline \multirow[t]{3}{*}{ NGC 4755-F } & $f_{1}, 4.88$ & 9.78 & 9.11 & 8.52 \\
\hline & $f_{2}, 4.54$ & 5.13 & 4.16 & 3.46 \\
\hline & $f_{3}, 5.27$ & 3.90 & 3.56 & 3.62 \\
\hline \multirow[t]{4}{*}{ NGC $4755-\mathrm{G}$} & $f_{1}, 6.63$ & $\overline{3.92}$ & 2.88 & 2.63 \\
\hline & $f_{2}, 6.42$ & 3.24 & 2.65 & 2.64 \\
\hline & $f_{3}, 6.15$ & 2.35 & 1.96 & 2.64 \\
\hline & $f_{4}, 7.28$ & 1.32 & 1.45 & 0.97 \\
\hline \multirow[t]{2}{*}{ NGC 4755-I } & $f_{1}, 5.59$ & 7.22 & 6.23 & 6.28 \\
\hline & $f_{2}, 7.83$ & 1.57 & 1.92 & 1.88 \\
\hline \multirow[t]{2}{*}{ NGC $4755-201$} & $f_{1}, 5.48$ & 6.65 & 5.32 & 5.14 \\
\hline & $f_{2}, 10.96$ & 1.10 & 0.99 & 1.21 \\
\hline \multirow[t]{3}{*}{ NGC 4755-202 } & $f_{1}, 6.29$ & 9.07 & 7.83 & 7.88 \\
\hline & $f_{2}, 0.31$ & 1.27 & 1.34 & 1.32 \\
\hline & $f_{3}, 9.24$ & 8.71 & 5.74 & 5.98 \\
\hline NGC 4755-210 & $f_{1}, 10.72$ & 3.84 & 3.44 & 3.65 \\
\hline NGC 4755-301 & $f_{1}, 7.66$ & 6.30 & 5.26 & 5.12 \\
\hline
\end{tabular}

and its first overtone. The residuals and the fit to the light curve indicate the presence of at least one more frequency at $f_{3}=5.85 \mathrm{~d}^{-1}$ (in the $U$-filter).

NGC 4755-202: Our observations resulted in the detection of 3 frequencies. We did not find the frequency $f=7.217 \mathrm{~d}^{-1}$ (Balona \& Koen, 1994).

NGC 4755-301: Our observations resulted in the detection a single frequency. For NGC 4755-113 and NGC 4755-307, we did not find any periodicities exceeding 3 mmag. In the near future, we will identify the modes using the fact that $\ell$ depends on the wavelength.

Acknowledgments. I am grateful to G. Handler, M. Hempel, and P. Mittermayer for their help in the gathering of the data.

\section{References}

Arp, H. \& van Sant, C.T. 1958, AJ, 63, 340

Balona, L.A., Dziembowski, W.A., \& Pamyatnykh, A.A. 1997, MNRAS, 289, 25

Balona, L.A. \& Koen, C. 1994, MNRAS, 267, 1071

Balona, L.A. 1994, MNRAS, 267, 1060

Breger, M., Zima, W., Handler, G., et al. 1998, A\&A, 331, 271

Koen, C. 1993, MNRAS, 264, 165

Kjeldsen, H. \& Frandsen, S. 1992, PASP, 104, 413

Moskalik, P. \& Dziembowski, W.A. 1992, A\&A, 256, L5

Sperl, M. 1996, DSSN, 10, 5

Stankov, A., Breger, M., Ashley, M.C.B., \& Prouton, 0. 1998, Ap\&SS, 260, 281 